\title{
Die Interessen der Pflege vertreten
}

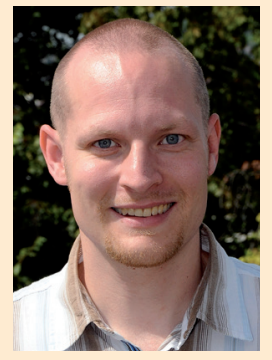

Christian Schäfer, Stuttgart
Welche Organisationsform könnte die Interessen der Pflege gegenüber der Gesellschaft und Politik am besten vertreten - eine Pflegekammer, eine sog. „Pflegekammer light“ oder doch gar nichts davon? Dass die Interessen der Pflege vertreten werden müssen bzw. sollten, wenn sich irgendwann einmal mehr als nur das minimal Notwendige in der Pflegepolitik ändern soll, wird immer klarer und ist nur noch schwer von der Hand zu weisen. Somit scheidet eigentlich all das aus, was zu unwirksam ist - sprich: Sich nicht oder wenig zu organisieren, ist auf Dauer keine Option. Fakt ist, dass die Bundesländer das Thema komplett unterschiedlich angehen - so ist z. B. in Rheinland-Pfalz die Pflegekammer seit der Gründung am 05.01.2015 Realität, in Hamburg etwa wurde nach einer Befragung von Pflegekräften, bei der die Mehrheit eine Pflegekammer ablehnte, das Projekt nicht weiter verfolgt.

Im Februar dieses Jahres hatte Bayerns Gesundheits- und Pflegeministerin Melanie Huml (CSU) für ihr Bundesland die Idee zur „Pflegekammer light“ - dies wäre eine Körperschaft öffentlichen Rechts, in der Pflegekräfte und -verbände freiwillig Mitglied werden können. Das ist auch ein entscheidender Hauptunterschied zur klassischen Pflegekammer, bei der neben dem Entrichten von Beiträgen auch die Mitgliedschaft Pflicht ist. Diese letztgenannte Eigenschaft einer „richtigen“ Pflegekammer scheint gleichzeitig auch der wichtigste Punkt zu sein, der einige Pflegekräfte so sehr stört, dass sie diese ablehnen. Huml will mit der abgespeckten Variante einen Kompromiss wagen, um zumindest einige Eckpunkte einer Kammer zu etablieren - diese wären z.B. die Mitwirkung an Gesetzgebungsvorhaben, die Weiterentwicklung der Qualität der Pflege und staatliche Vollzugsaufgaben (wie Bewilligungen nach der Förderrichtlinie in der Altenpflege). Huml forderte beim zweiten runden Tisch „Pflegekammer“ (eine Arbeitsgruppe aus unabhängigen Experten, Befürwortern und Kritikern einer Pflegekammer) die Beteiligten auf, dem Konzept „Pflegekammer light“ bis Anfang März zuzustimmen.

Der Präsident des Deutschen Pflegerats (DPR) Andreas Westerfellhaus ist allerdings von der Light-Variante nicht sehr begeistert. So hat laut Westerfellhaus eine repräsentative, vom Ministerium selbst in Auftrag gegebene Umfrage unter Pflegekräften in Bayern ergeben, dass sich die Hälfte der Befragten für eine Pflegekammer aussprechen. Zudem sei „die Verweigerung einer Pflegekammer [...] ein Beweis für die Konzeptlosigkeit und kommt einem Kniefall vor deren Gegnern gleich", so Westerfellhaus. Eine Körperschaft öffentlichen Rechts könne außerdem die Interessen der Fachpflegekräfte nicht mit der ihnen gebührenden Schlagkraft vertreten, wie dies eine Kammer tun könne.

Zusammenfassend gibt es für den oben geschilderten Sachverhalt Vor- und Nachteile, die je nach Gewichtung mal für die „Pflegekammer light“ (z.B. Vorteil der wahrscheinlich breiteren Akzeptanz unter Pflegekräften) und mal für die klassische Pflegekammer (z.B. Vorteil der größeren Schlagkraft) sprechen. Was aber immer mehr durchscheint ist, dass beide Pflegekammerversionen für sich gesehen wohl besser für Pflegekräfte sind, als nichts davon.

Ähnliches gilt für die Antikoagulation - hier gibt es Vor- und Nachteile bzgl. der einzelnen Substanzen, natürlich auch abhängig vom individuellen Patienten. Sicher ist aber, dass eine Hemmung der Blutgerinnung aufgrund des extrakorporalen Blutkreislaufs bei der Hämodialysebehandlung notwendig ist. Um in das recht unübersichtliche Thema etwas Struktur zu bringen, haben die beiden Gasteditoren Prof. Sylvia Stracke und Dr. Christian Aymanns, Greifswald, zusammen mit mir das vorliegende Schwerpunktheft der Dialyse aktuell geplant. Ich wünsche Ihnen eine erhellende Lektüre der aktuellen Beiträge zur Antikoagulation in der Nephrologie ab Seite 74 ! 